\title{
Neisseria gonorrhoeae Screening
}

National Cancer Institute

\section{Source}

National Cancer Institute. Neisseria gonorrhoeae Screening. NCI Thesaurus. Code C92298.

Examination of a biological specimen to detect the presence of Neisseria gonorrhoeae. 